\title{
ERRATUM
}

\section{On the Solution of the Michaelis-Menten Equation}

\author{
Stuart L. Beal ${ }^{1}$ \\ Received May 19, 1982
}

Subsequent to the recent appearance of my article in $J P B$, Vol. 10, No. 1, pp. 109-119, in which a solution to the Michaelis-Menten equation is presented, Mr. W. D. Robinson has informed me that this same solution has appeared earlier in the article, "Nonlinear problems in statistical and mathematical interpretation," by J. P. R. Toothill, and published in Progress in Industrial Microbiology, Vol. 2, by Heywood and Company Ltd., London, in 1960. A reader of that article will find that the symbol $\phi$ is used to denote the function, $F$, and he should also be aware that in the early part of Section 2.4, where this function is introduced, there is an unfortunate typographical error: the product, ket, should be divided, wherever it occurs, by $K$. Values of this function are tabled in the article for values of the argument on the grid $-1.49(0.01) 5.99$. Table I of my article uses the grid $-7.00(0.01) 7.84$. However, Toothill's values of the function are given to 6 digits after the decimal point, whereas my values are given to only 3 significant figures.

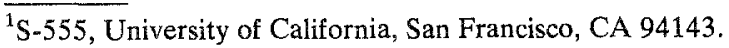

